\title{
Embolismo pulmonar masivo y trombo en ventrículo derecho postinfarto agudo de miocardio
}

\section{Massive pulmonary embolism and right ventricle thrombus post-acute myocardial infarct}

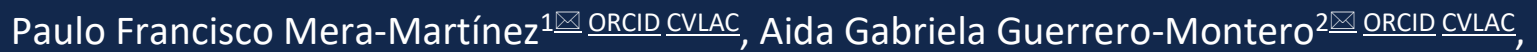

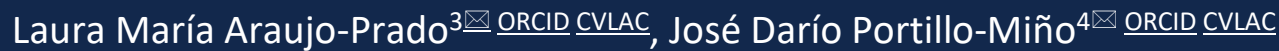

${ }^{1}$ Residente de Cirugía General, Universidad Nacional de Colombia, Bogotá D.C.

${ }^{2}$ Médico, Facultad de Ciencias de la Salud, Universidad Cooperativa, Pasto. Colombia.

${ }^{3}$ Facultad de Ciencias de la Salud, Fundación Universitaria San Martín, Pasto. Colombia. Departamento de Especialidades Quirúrgicas, Fundación Hospital San Pedro, Pasto. Colombia.

${ }^{4}$ Facultad de Ciencias de la Salud, Fundación Universitaria San Martin, Pasto. Colombia. Grupo de Investigación GINFYCA, Fundación Hospital San Pedro, Pasto. Colombia

Fecha correspondencia:

Recibido: octubre 12 de 2020 Revisado: mayo 03 de 2021.

Aceptado: mayo 13 de 2021.

Forma de citar:

Mera-Martínez PF, GuerreroMontero AG, Araujo-Prado LM, Portillo-Miño JD. Embolismo pulmonar masivo y trombo en ventrículo derecho postinfarto agudo de miocardio. Rev CES Med. 2021; 35(2): 165-174 10.21615/cesmedicina.5908

Open access

(C) Derecho de autor

Licencia creative commons

Ética de publicaciones

Revisión por pares

Gestión por Open Journal System DOI: http://dx.doi.org/10.21615/

cesmedicina.5908

ISSNe 2215-9177

ISSN 0120-8705

Publica con nosotros

\section{Resumen}

Introducción: un trombo en ventrículo derecho y embolismo pulmonar post infarto agudo de miocardio sin elevación del ST es muy inusual. Es considerado una emergencia médica y es imperativo instaurar una opción terapéutica inmediata. Presentación del caso: paciente femenina de 80 años quien consultó en sala de emergencias por síncope, dolor torácico y disnea persistente. Se confirmó síndrome coronario agudo por electrocardiograma y troponina I elevada. En estudios complementarios se encontró hallazgo incidental de trombo en el ventrículo derecho y un angio-TAC confirmó embolismo pulmonar masivo. Discusión: el trombo en ventrículo derecho y embolismo pulmonar masivo post infarto es un fenómeno muy raro en el escenario clínico, debido a la instauración temprana de la terapia anti-isquémica. Es necesario mayor investigación para aclarar este fenómeno.

Palabras clave: Infarto del miocardio sin elevación del ST; Ventrículo cardiaco; Trombo, embolismo pulmonar. 


\section{Abstract}

Introduction: right ventricular thrombus and pulmonary embolism after acute myocardial infarction without ST elevation is very unusual. It is considered a medical emergency and it is imperative to establish an immediate therapeutic option. Case presentation: An 80-year-old female patient visited the emergency room for syncope, chest pain, and persistent dyspnea. Acute coronary syndrome was confirmed by electrocardiogram and elevated troponin I. In complementary studies, an incidental finding of a thrombus in the right ventricle was found and a CT angiography confirmed massive pulmonary embolism. Discussion: thrombus in the right ventricle and massive post-infarction pulmonary embolism is a very rare phenomenon in the clinical setting due to the early introduction of anti-ischemic therapy. More research is needed to clarify this phenomenon.

Keywords: Non-ST-Elevated Myocardial Infarction; Pulmonary embolism; Heart ventricle.

\section{Introducción}

El infarto de miocardio sin elevación del ST -NSTEMI- (non-ST elevación myocardial infarction; por sus siglas en inglés) es una enfermedad con alta carga económica. La mediana de edad al momento de presentación del síndrome coronario agudo es de 68 años y los hombres superan en número a las mujeres con una razón de $3: 2^{(1)}$. La incidencia de síndrome coronario agudo en Estados Unidos superan los 780000 y de ellos, aproximadamente el $70 \%$ tendrá NSTEMI ${ }^{(1)}$.

El trombo del ventrículo derecho y embolismo pulmonar post-NSTEMI se conocen de forma insuficiente, puesto que es muy rara su presentación en el escenario clínico. Se ha detectado un trombo en ventrículo derecho cerca del $4,5 \%$ de los pacientes con embolismo pulmonar y sometidos a ecocardiografía ${ }^{(2)}$.

La presentación del trombo en ventrículo derecho está asociada a fibrilación auricular, enfermedad cardiaca estructural o catéter in $\operatorname{situ}^{(3)}$. Probablemente, el trombo proviene del sistema venoso pulmonar, se encuentra asociado a alta mortalidad y su hallazgo se realiza de forma incidental en la autopsia. Es considerada una emergencia médica, lo que hace imperativo instaurar una opción terapéutica de inmediato. Por lo que un trombo en ventrículo derecho y el embolismo pulmonar representan un verdadero dilema en el manejo terapéutico ${ }^{(4)}$.

Se presenta el caso de una paciente con trombo en ventrículo derecho y embolismo pulmonar masivo post-NSTEMI a pesar de terapia anti isquémica, anticoagulante y antiagregante, el cual es un fenómeno muy extraño en el escenario clínico. 


\section{Presentación de caso}

Una mujer de 80 años acudió al servicio de emergencias por dolor torácico opresivo que se extendía al miembro superior izquierdo, asociado a disnea, tos seca no productiva y posteriormente, pérdida de la conciencia por cinco minutos, más desorientación y disartria. Entre los antecedentes de importancia se destacaba enfermedad pulmonar obstructiva crónica oxígeno dependiente, hipertensión arterial controlada con losartán y artritis reumatoide en manejo con azatioprina.

Al momento de la admisión se encontró una paciente hemodinámicamente estable, afebril, con ingurgitación yugular grado II, ruidos cardiacos rítmicos, no soplos a la auscultación, ruidos respiratorios disminuidos, estertores en ambas bases pulmonares, extremidades con deformidad interfalángica en manos, sin edemas. En sistema nervioso central: alerta, desorientada, reflejos profundos aumentados, pupilas isocóricas reactivas, no signos de focalización, lenguaje adecuado y coherente, escala de coma de Glasgow 13/15.

Al ingreso, los exámenes de sangre mostraron una función renal y hepática conservada; sin alteración electrolítica, sin leucocitosis o neutrofilia. Se realizó electrocardiograma que evidenció infarto agudo de miocardio sin elevación del ST más troponina I de alta sensibilidad alterada (> percentil 99). Se realizó impresión diagnóstica de infarto agudo de miocardio sin elevación del ST, por el cual se inició terapia anti-isquémica, tratamiento anticoagulante pleno y antiagregación dual. Fue valorada por Medicina Interna que solicitó ecocardiograma para estudio de sincope, probablemente de origen cardiogénico y daño cardiaco estructural y medición troponina de control.

Es valorada por el servicio de Cardiología, que mediante ecocardiograma transtorácico, identifica una imagen de densidad de tejidos blandos, móvil, con un diámetro de 24 x $11 \mathrm{~mm}$, adherido a cuerdas tendinosas en la válvula tricúspide más hipocinesia septal severa, compatible con trombo intracavitario derecho (figura 1). Ante estos hallazgos se sugiere evaluar presunta etiología pulmonar. Se llevó a cabo angiotomografía axial computarizada en la que se observó trombo que ocluye el flujo sanguíneo de arteria pulmonar derecha (figura 2).

Dado que la paciente tenía un NSTEMI, lo cual era una contraindicación para iniciar trombolisis y que se instauró terapia anti-isquémica, antiagregante y anticoagulante; a pesar de ello, fue paradójico descubrir un trombo ventricular derecho y embolia pulmonar. Por esta razón, para la disolución del trombo, se administró warfarina. 
En los siguientes días un ecocardiograma de control evidenció ausencia del coagulo en cavidad ventricular derecha y de la obstrucción de arteria pulmonar derecha y arterias segmentarias. La paciente evolucionó y en esas condiciones se da el egreso hospitalario anticoagulado con warfarina y control ambulatorio.

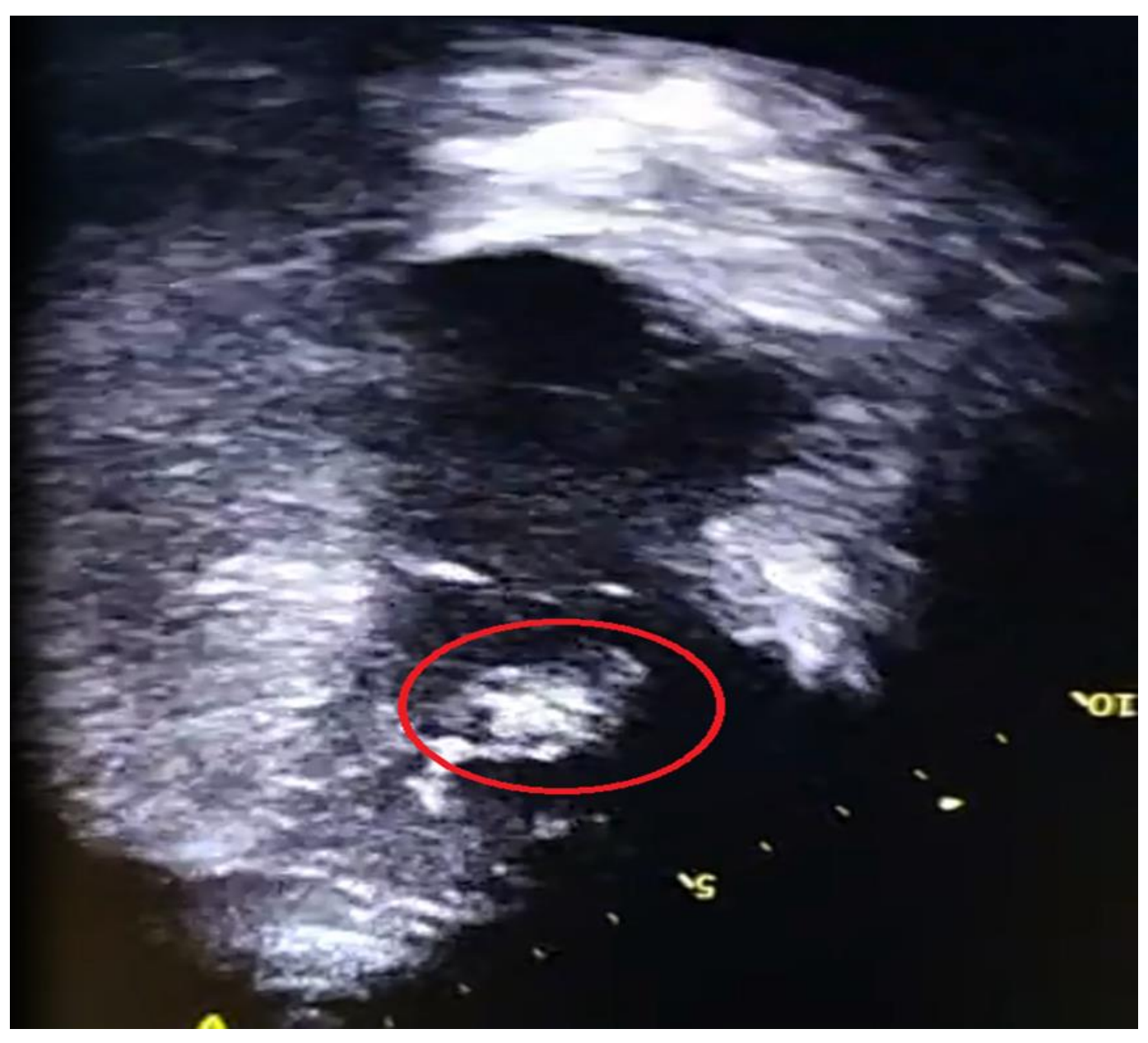

Figura 1. Ecocardiograma.

Imagen sugestiva de lesión hiperecóica en ventrículo derecho, con presencia de trombo único adherido del velo septal de la válvula tricúspide con trombo con un tamaño de 24 x $11 \mathrm{~mm}$ más insuficiencia tricúspidea moderada. 


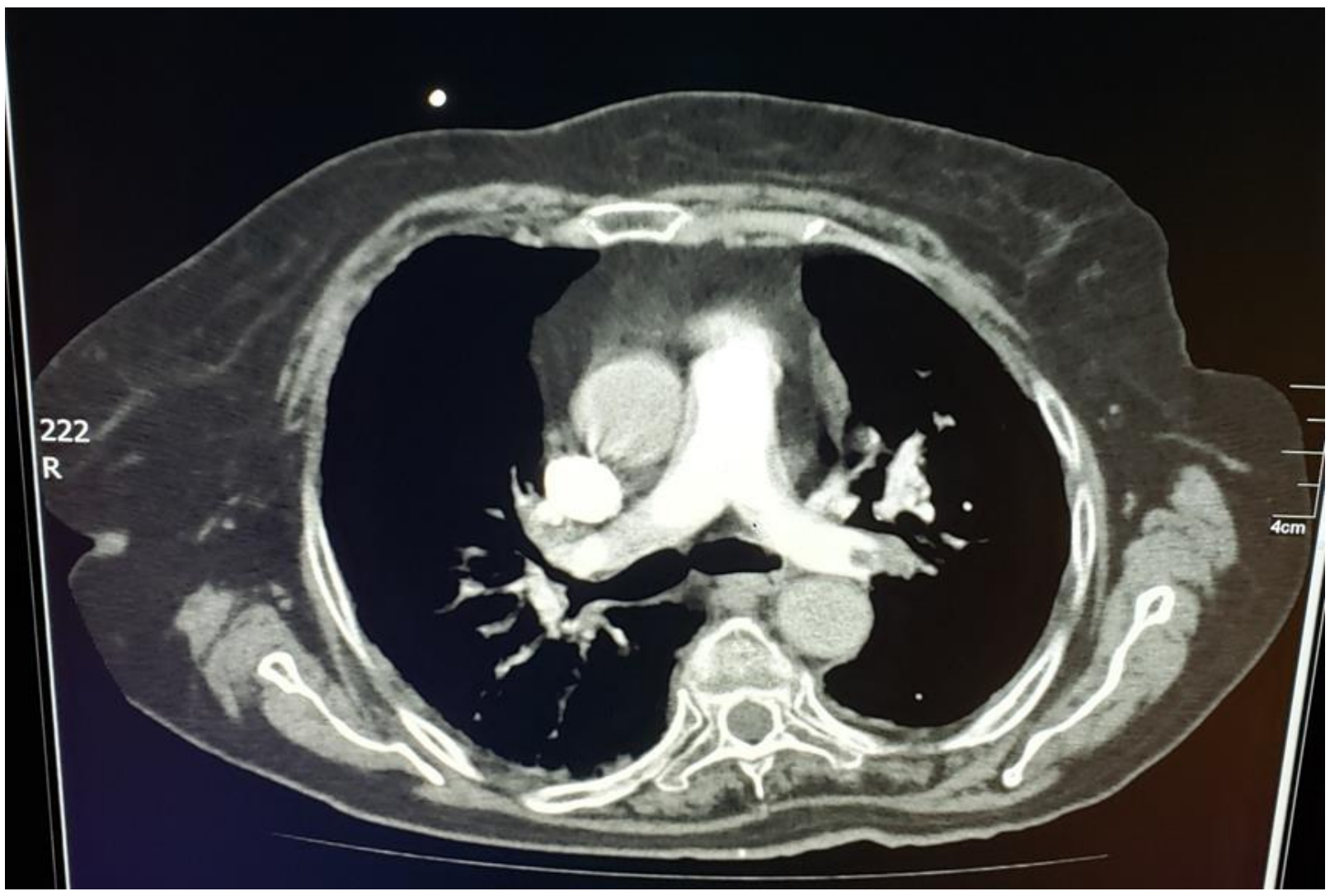

Figura 2. AngioTAC de tórax.

Área hipodensa con defecto de llenado por trombo que ocluye el flujo sanguíneo dentro de la arteria pulmonar derecha más opacificación de las ramas principales y de las arterias segmentarias para ambos lóbulos inferiores comprometiendo los segmentos mediales, laterales y basales.

\section{Discusión}

El trombo en ventrículo derecho y embolismo pulmonar predicen una condición catastrófica con alta mortalidad. En un reciente estudio estiman una mortalidad alrededor del $27 \%{ }^{(5)}$ y Vrey et al. reportan una mortalidad que alcanza hasta $44 \%{ }^{(6)}$. En esas circunstancias, el diagnóstico y tratamiento debe iniciarse lo más pronto posible con el fin de evitar ese desenlace.

Es preciso comprender esta entidad puesto que expone un escenario desafiante en la práctica clínica para el abordaje diagnóstico y terapéutico ${ }^{(4)}$. Al respecto, se proponen algunas teorías sobre la etio-patogénesis: en primer lugar, el embolismo pulmonar puede enmascararse como un infarto agudo de miocardio, aunque es un fenómeno excepcional en la era de la heparina como pilar fundamental en el tratamiento del infarto. En segundo lugar, considerar el 
embolismo pulmonar como una complicación del infarto que podría darse en casos extraños como la presentación o terapia de revascularización tardía(7). En este orden de ideas se ha contemplado que el desarrollo de un trombo en el ventrículo derecho sea debido a un miocardio acinético que produce estasis sanguínea y conduce a la formación del trombo con posterior embolización hacia la vasculatura pulmonar ${ }^{(8)}$. Este postulado es bastante sugestivo con lo ocurrido en la paciente, quien presentó trombo en ventrículo derecho y embolismo pulmonar masivo post-NSTEMI; además, en el ecocardiograma se logró constatar la hipocinesia septal.

Boukantar et al. presentan un caso similar de embolismo pulmonar con trombo en ventrículo derecho post-NSTEMI ${ }^{(9)}$. Igualmente, se han reportado algunos casos de infarto agudo de miocardio asociado a embolismo pulmonar masivo en foramen ovale permeable ${ }^{(10)}$, trombo en ventrículo derecho y embolismo pulmonar masivo debido a un defecto atrial septal ${ }^{(11)}$, trombo en ventrículo derecho asociado a síndrome de Behçet ${ }^{(12)}$ y síndrome nefrótico ${ }^{(13)}$. El trombo en ventrículo derecho ha sido escasamente documentado, observándose en el 4-18 \% de los pacientes con embolismo pulmonar masivo ${ }^{(14)}$. Según Casazza et al., el trombo en ventrículo derecho y embolismo pulmonar solo ha sido detectado mediante el ecocardiograma en el $4,5 \%{ }^{(2)}$.

Los trombos son comunes en las aurículas puesto que hay mayor estasis del flujo sanguíneo. Las alteraciones en las proteínas que afectan el sistema hemostático, tales como la deficiencias de la antitrombina III, el déficit o la resistencia a la proteína $\mathrm{C}$, el déficit de la proteína $\mathrm{S}$, la mutación del gen de la protrombina (factor II), las mutaciones de la metiltetrahidrofolato reductasa, el factor $V$ de Leiden y la protrombina $G 20210 A$, favorecen la formación del trombo en cavidades cardiacas ${ }^{(15)}$.

Desde el punto de vista morfológico, el trombo intracavitario se divide en dos tipos: los tipo A que tienen forma de gusano, son extremadamente móviles y en su mayoría representan coágulos venosos periféricos que se alojan temporalmente en el corazón derecho. Por su parte, los trombos tipo $B$ que son estructuralmente similares a los trombos tipo $A$, son menos móviles, se adhieren a la pared auricular o ventricular derecha y tienen una conexión de base amplia por el cual se desarrollan dentro del ventrículo derecho ${ }^{(11)}$. De acuerdo a la descripción ecocardiográfica, es posible que en el caso presentado corresponda un coagulo tipo A, móvil y con alto potencial de embolización. 
El trombo fácilmente puede ser confundido con otras estructuras fisiológicas o patológicas del corazón, siendo necesario diferenciarlas. Entre las anormalidades congénitas se encuentra la red de Chiari, aneurisma auricular septal y válvulas de Tebesio y Eustaquio persistentes. Por otro lado, las anomalías adquiridas se caracterizan por los tumores intracardiacos, vegetaciones y mixomas ${ }^{(16)}$.

La ecocardiografía es una herramienta esencial para investigar la localización del trombo. Los ecocardiogramas transtorácico y transesofágico son usados para detectar las anomalías cardiacas estructurales. Su rendimiento se destaca por ser de fácil acceso, no invasivos, ampliamente disponibles, no ocasionan demasiadas molestias y pueden ser tomado al borde de la cama del paciente ${ }^{(11)}$. La ecocardiografía puede evaluar la función ventricular derecha, realizar el control y seguimiento durante y después del tratamiento. El ecocardiograma en serie también es útil cuando el estado clínico se deteriora porque puede evidenciar trombos que no se detectaron en el examen inicial ${ }^{(11)}$.

Otras modalidades de imagen pueden caracterizar mejor la lesión en pacientes estables cuando el ecocardiograma no es concluyente. La resonancia magnética con contraste sirve en este escenario detectando trombos murales con una sensibilidad del $88 \%$ y especificidad del 99 $\%{ }^{(17)}$. Además, brinda información adicional de masas intracardiacas, tumores o vegetaciones, que requieren una intervención terapéutica distinta.

Entre las opciones terapéuticas en pacientes con embolismo pulmonar e inestabilidad hemodinámica está ampliamente aceptada la indicación de trombolisis ${ }^{(4)}$. En cuanto a los pacientes con contraindicación para trombolisis y sin mejoría clínica posterior a su administración, está recomendada la embolectomía. En los casos de embolismo pulmonar complicado con trombo en ventrículo derecho, la trombolisis o embolectomía deben ser considerados en ausencia de contraindicaciones en pacientes con un trombo tipo A, aunque se encuentre con estabilidad hemodinámica, pues se ha observado que existe una alta probabilidad de embolización y mortalidad ${ }^{(4)}$. En trombos tipo $B$ tiene un resultado más favorable y la trombolisis actualmente no está recomendada ${ }^{(11)}$. En este caso, debido a que se trataba de un coagulo tipo A, la terapia más adecuada era la trombolisis o la embolectomía; pero, como padeció NSTEMI existía contraindicación, por lo que se decidió anticoagulación oral. 
Mayo - agosto de 2021

\section{Conclusiones}

Hoy en día, el trombo en ventrículo derecho y embolismo pulmonar masivo post-NSTEMI es muy extraño debido al tratamiento anti isquémico, anticoagulante y antiagregante instaurado para el síndrome coronario agudo. Es una condición clínica con alta mortalidad, por el cual es necesario fomentar la sospecha clínica teniendo en cuenta que es una enfermedad de presentación inusual.

\section{Conflicto de Interés}

Los autores no declaran.

\section{Consentimiento informado}

Se obtuvo el consentimiento informado para la publicación de este reporte de caso y las imágenes correspondientes. El consentimiento reposa en poder de los autores.

\section{Financiación}

Los autores.

\section{Bibliografía}

1. Basit H, Malik A, Huecker MR. Non-ST segment elevation (NSTEMI) myocardial infarction. [Updated 2020 May 4]. StatPearls Internet Treasure Isl FL StatPearls Publ 2020 Jan[Internet]. Disponible en: Available from: https://www.ncbi.nlm.nih.gov/books/NBK513228/

2. Casazza F, Becattini C, Guglielmelli E. Prognostic significance of free-floating right heart thromboemboli in acute pulmonary embolism: results from the Italian Pulmonary Embolism Registry. Thromb Haemost. 2014;111:53-7.

3. Naeem K. Floating thrombus in the right heart associated with pulmonary embolism: The role of echocardiography. Pak J Med Sci. 2015;31(1):233-235.

4. Mujer M, Saleh Y, Abro C, Kandola SK. M. Pulmonary embolism with right ventricular thrombus: a management dilemma. BMJ Case Rep. 2019;12(2):e229184.

5. Rose PS, Punjabi NM, Pearse DB. Treatment of right heart thrombo-emboli. Chest. 2002;121:806-814. 
6. De Vrey EA, Bax JJ, Poldermans D. Mobile right heart thrombus and massive pulmonary embolism. Eur J Echocardiogr. 2007;8:229-231.

7. Kuno T, Imaeda S, Hashimoto K, Ryuzaki T, Saito T, Yamazaki H, Tabei R, Kodaira M, Hase $M$, and Numasawa $Y$. Recent inferior myocardial infarction complicated with a right ventricular thrombus detected by three cardiac imaging modalities. Intern Med. 2018;57(5), pp.693-695.

8. Al-Sadawi $M$, Abdul R, McFarlane Sl. Right ventricular infarction associated with pulmonary embolism. Am J Med Case Rep. 2019;7(7):151-157.

9. Boukantar M, Lim P, Mitchell-Heggs L. Right ventricular thrombus and pulmonary embolism in patient with anterior myocardial infarction. Eur Heart J. 2010;31(23):28702870.

10.Zimmermann FM, Peels KH. Simultaneous massive pulmonary embolism and acute myocardial infarction, associated with patent foramen ovale. Eur Heart J. 2014;7;35(30):2046.

11. Dinesh Kumar US, Nareppa U, Shetty SP, et al. Right ventricular thrombus in case of atrial septal defect with massive pulmonary embolism: a diagnostic dilemma. Ann Card Anaesth. 2016;19:173-6.

12. Payam Ebrahimifar, Javad Shahabi. Right ventricular thrombosis as a manifestation of Behçet's syndrome. ARYA Atheroscler. 2017;13(2):91-94.

13. Lempp S, Schwenger V. Isolated right ventricular thrombus in an adult patient with nephrotic syndrome: a case report. J Med Case Rep. 2017;11(1):311.

14. Torbicki A, Galié N, Covezzoli A, Rossi E, De Rosa M, Goldhaber SZ. Right heart thrombi in pulmonary embolism:results from the International Cooperative Pulmonary Embolism Registry. J Am Coll Cardiol. 2003;41:2245-2251.

15. Torres-Martel J.M., Bautista-Hernández K.M. Trombofilia hereditaria y trombo intracardíaco. Rev Col Cardiol. 2018;25(3):238.e1---238.e4.

16. Verma R, Duncanson ER, Bajpai A. Right atrial thrombus arising from the junction of the right atrium and the inferior vena cava. Cardiovasc Pathol. 2014;23(5):317-318. 
17.Tsang BK, Platts DG, Javorsky $G$, et al. Right ventricular thrombus detection and multimodality imaging using contrast echocardiography and cardiac magnetic resonance imaging. Heart Lung Circ [Internet]. 2012;21:185-8. Disponible en: doi:10.1016/j.hlc.2011.08.012 